\title{
Bidimensional scattering of TM and TE polarized waves by dielectric bodies using the CG-FFT method: Application to mobile communications systems
}

\author{
M. Vaquer, J.J. Mallorqui, A. Cardama, \\ L. Jofre
}

Grup Antenes-Microones-Radar, Dept. Teoria del Senyal i Comunicacions. ETSETB, Universitat Politècnica de Catalunya. P.O. Box 30002,08080 Barcelona, SPAIN.

\section{Abstract}

In the study of electromagnetic radiation effects over dielectric bodies, effective numerical methods are needed. At present, there is a lack of tools for measuring this effects and due to the huge growth of microwave devices, like cellular radio telephones working close to the human body, it is necessary to develop accurate numerical methods for evaluating those effects. One of the most efficient and extensively used method is the FFT-CGM (Fast-Fourier-Transform Conjugate Gradient Method). In this paper, algorithms for solving the two-dimensional scattering of transversal magnetic (TM) and transversal electric (TE) polarized waves by a lossy dielectric and metallic objects are presented. At the same time, the SAR (Specific Absorption Rate) of tissues and the modified radiation diagrams when the body is present are obtained from the diffracted fields.

\section{Introduction}

The development of biological applications in electromagnetics and the study of the radiation effects require fast and accurate methods for the computation of electromagnetic fields inside inhomogeneous lossy dielectrics exposed to a known incident field. The case of metallic structures is interesting too. Analytical solutions exist only for simple geometries such as the cylindrical and the spherical ones. Those solutions are mainly used to check the accuracy and behaviour of numerical results.

Scattering by inhomogeneous lossy dielectric and metallic bodies can be solved by using global or local techniques. In this paper the global ones, that use wave expansion functions and the integral equation over the domain of the object, have been used. Approaches for the 2-D TM and TE polarized cases have been implemented and tested.

\section{Description of the Algorithms}

In the TM case the integral equation can be expressed as

$$
\vec{E}^{i}(\vec{r})=\vec{E}(\vec{r})-\vec{E}^{s}(\vec{r})=\vec{E}(\vec{r})+k_{0}^{2} \int_{s^{\prime}} \frac{\varepsilon_{0}-\varepsilon(\vec{r})}{\varepsilon_{0}} \vec{E}(\vec{r}) G\left(\left\|\vec{r}-\vec{r}^{\prime}\right\|\right) d s^{\prime}
$$

with $\overrightarrow{\mathrm{E}}(\overrightarrow{\mathrm{r}})$, the incident field, $\overrightarrow{\mathrm{E}}(\overrightarrow{\mathrm{r}})$, the diffracted field and $\overrightarrow{\mathrm{E}} \mathrm{s}(\overrightarrow{\mathrm{r}})$, the scattered field. The Green's function, $G(\|\vec{r}-\vec{r}\|)$, is given by

$$
G\left(\left\|\vec{r}-\vec{r}^{\prime}\right\|\right)=\frac{1}{4 j} H_{0}^{(2)}\left(k_{0}\left\|\vec{r}-\vec{r}^{\prime}\right\|\right)
$$

Equation (1) can be solved by using the method of moments with pulse expansion functions and point matching, Richmond [1]. This method reduces the integral equation to a linear system and requires a matrix inversion, that can be optimized in computational time and memory storage by using the FFT algorithm and the iterative CGM, Borup and Gandhi [2].

In the TE case, the integral equation is expressed in terms of the electric flux rather than the electric field because it is continuous at the discontinuity interface between media of different permittivities

$$
\vec{E}^{i}(\vec{r})=\frac{\vec{D}(\vec{r})}{\varepsilon(\vec{r})}+\left(k_{0}^{2}+\operatorname{graddiv}\right) \int_{s^{\prime}} \frac{\varepsilon_{0}-\varepsilon(\vec{r})}{\varepsilon_{0}} \frac{\vec{D}(\vec{r})}{\varepsilon(\vec{r})} G\left(\left\|\vec{r}-\vec{r}^{\prime}\right\|\right) d s^{\prime}
$$

A discretization in the spatial domain by means of a staggered grid is firstly introduced. Then, the vector equation is tested with rooftop functions and a suitable expansion of the vector potential in this equation is done. Once again, the two-dimensional FFT algorithm and the $\mathrm{CG}$ method can be used to solve the linear operator more efficiently, Zwamborn and Van der Berg [3].

In the presence of metallic bodies, it is necessary to express the former equations in terms of the density current in order to avoid singularities in the integral equations in both TM and TE cases. Density currents should be used over the metallic surfaces but it is not possible to do so, owing to the discretization introduced, Joachimowicz and Pichot [4]. Thus, real scattered fields are obtained from non-physical volumetric density currents defined on each cell of the body. For instance, in the TM case

$$
\vec{E}^{i}(\vec{r})=\frac{\vec{J}(\vec{r})}{\varepsilon_{0}-\varepsilon(\vec{r})}+k_{0}^{2} \int_{s^{\prime}} \frac{J(\vec{r})}{\varepsilon_{0}} G\left(\left\|\vec{r}-\vec{r}^{\prime}\right\|\right) d s^{\prime}
$$

This equation is only valid inside the body, because in the outside medium the term $1 /(\varepsilon 0-\varepsilon(r))$ yields to a singularity. Moreover, $\vec{E} \cdot(\vec{r})=-\vec{E}(\vec{r})$ must be forced into the metal where the total field has to be zero.

\section{Specific Absorption Rate (SAR)}

One way of measuring the power effects of the electromagnetic radiation inside biological bodies is the SAR (Specific Absorption Rate). Because of the power absorption distribution is highly dependent on electrical conductivity levels in tissues, the SAR is a function of the conductivity, the density and the electrical field intensity

$$
S A R=\frac{1}{2} \frac{\sigma\|E\|^{2}}{\rho} W \cdot \mathrm{kg}^{-1}
$$

With good discretized models of biological bodies, the effects of radiation inside them, for instance the dissipation power dissipated by cellular phones in the head, can be evaluated, in terms of SAR, from diffracted fields.

\section{Radiation Patterns}

An important effect of the presence of bodies near antennas is the modification of its radiation diagram. In this paper, a weak form for obtaining them is presented. The expression of the distant diffracted field, which can be directly associated with the radiation pattern, can be expressed in terms of the diffracted field in each cell of the discretization grid

$$
\vec{E}(\vec{r})=\vec{E}^{i}(\vec{r})-k_{0}^{2} \int_{s^{\prime}} \frac{\varepsilon_{0}-\varepsilon(\vec{r})}{\varepsilon_{0}} \vec{E}(\vec{r}) G^{\prime}(\|\vec{r}-\vec{r}\|) d s^{\prime}
$$

The term $E(\vec{r})$ is the normal component of the diffracted field in the TM case, or the $\phi$ component of the diffracted field in the TE case, the only components that affect the far field. $G^{\prime}\left(\left\|\vec{r}, \vec{r}^{\prime}\right\|\right)$ is the Green's function using the asymptotic form of the Hankel function for large arguments. 
In both TM and TE cases, the FFT cannot be implemented because the convolution involves points inside and outside the body.

\section{Numerical Results}

Numerical results for both the TM and TE cases are presented. For the TM case a two-layered dielectric and metallic core cylinder has been studied. The inner layer (radius $1.4 \mathrm{~cm}$ ) is metallic, the intermediate one (radius $3.0 \mathrm{~cm}$ ) is a dielectric with a permittivity of $5.0 . j 8.3$ and the outer one (radius $4.8 \mathrm{~cm}$ ) has a permittivity of $26.7-\mathrm{j} 0.1$. The external medium is water with a permittivity of 78.6-j10.5. The emitter antenna is placed at $15.0 \mathrm{~cm}$ and a circular receiver array at $10.0 \mathrm{~cm}$. A discretization of $0.1 \lambda$ has been used. Fig. 1 shows two radial cuts of the diffracted field inside the cylinder in comparison with the theoretical ones. Fig. 2 shows the scattered field in a circular grid of 64 points around the body compared with the exact solution. The incident field is a 2 $\mathrm{GHz}$ cylindrical wave generated by a current of $1 \mathrm{~A} / \mathrm{m}$.

For the TE case a two-layered dielectric cylinder has been studied and radial cuts of the total field are presented in Figs.3 and 4. The inner layer (Mdius $9.4 \mathrm{~cm}$ ) has a permittivity of 5.7 j2.99 and the outer (radius $15.0 \mathrm{~cm}$ ) $54-\mathrm{j} 83.92$. The incident wave is a $300 \mathrm{MHz}$ plane wave of $1 \mathrm{~V} / \mathrm{m}$ and the external medium is the vacuum. A discretization of $0.1 \lambda$ has been used too.

One SAR image of a human head is included in Fig. 5. The incident field is a $1.89 \mathrm{GHz}$ cylindrical wave generated by a current of $3 \mathrm{~mA} / \mathrm{m}$ with lateral incidence and placed at $3.0 \mathrm{~cm}$ from the ear. Fig. 6 shows the magnitude of head tissues permittivity used in the numerical model.

The experimental measurement of the radiation pattern of a short monopole antenna in the presence of a phantom human head is presented in Fig. 7, Torres et al.[5]. The same radiation pattern is computed with the TM algorithm described above and the results are shown in Fig. 8. The main difference is in the opposite side of the antenna because in the simulated case, and with the bidimensional approximation, the head has been modeled as an infinite length cylinder with the section of the real head, producing a higher attenuation in the radiated fields. In the anechoic chamber measurement the phantom is not infinite and, consequently, the attenuation introduced is lower. The radiation pattern has a gain of about $4.0 \mathrm{~dB}$ in the antenna side in both cases.

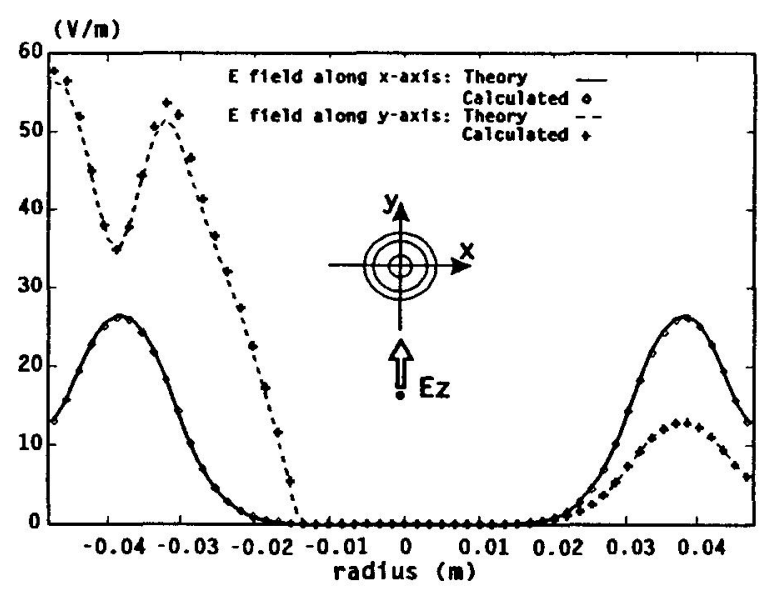

Fig. 1 Radial cuts of the electric field in the TM case.

\section{Conclusions}

Effective algorithms for computing the fields for the TM and TE cases inside lossy dielectric and metallic bodies are presented yielding excellent agreement with the analytical results. Moreover, tools for evaluating SAR and radiation diagrams are obtained. There are some limitations in the validity of the diagrams due to the bidimensional approximation employed. This bidimensional algorithms are in the process of extension to the 3 $D$ case and would be a useful tool for the mobile communications antenna design and for the study of power dissipation inside biological tissues. Another future direction is the modelling of the antenna and its feed, because at this moment it has been supposed that the near-field radiation of the antenna is not affected by the presence of the human head.

\section{Acknowledgement}

This work has been sponsored by the Comision Interministerial de Ciencia y Tecnología (CICYT) under Grant TIC 91/1034.

\section{References}

[1] J.H. Richmond, "Scattering by a dielectric cylinder of arbitrary cross section," IEEE Trans. on Antennas and Propagation, Vol. AP-13, pp. 334-341, Mar. 1965.

[2] D.T. Bourup, O.P. Gandhi, "Calculation of High-Resolution SAR Distributions in Biological Bodies Using the FFT Algorithm and Conjugate Gradient Method," IEEE Trans. on Microwave Theory and Techniques, Vol. MTT-33, pp. 417-419, May 1985.

[3] P. Zwamborn, P.M. van den Berg, "A Weak Form of the Conjugate Gradient FFT Method for Two-Dimensional TE Scattering Problems," IEEE Trans. on Microwave Theory and Techniques, Vol MTT-39, pp. 953-960, Jun. 1991.

[4] N. Joachimowicz, C. Pichot, "Comparison of Three Integral Formulations for the 2-D TE Scattering Problem," IEEE Trans. on Microwave Theory and Techniques, Vol. MTT-38, pp. 178185, Feb. 1990.

[5] J. Torres, J. Mallorqui, J. de Mora, S. Blanch, J. Romeu, A. Cardama, "Diseño y Medida de Antenas para un Equipo Portátil a $1.89 \mathrm{GHz}$, y su Interacción con el Cuerpo Humano". Actas del VII Simposium nacional de la URSI, pp. 1103-1107, Sept. 1992.

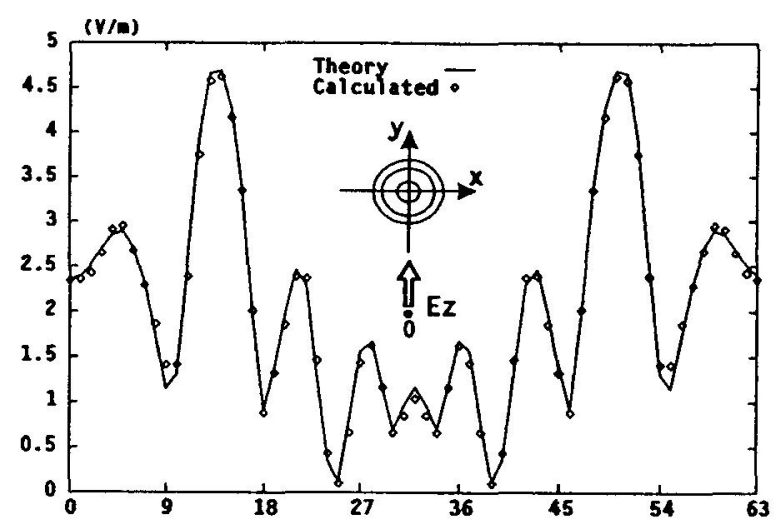

Fig. 2 Scattered field around the object in the TM case for an incident cylindrical wave originating in 0 . 


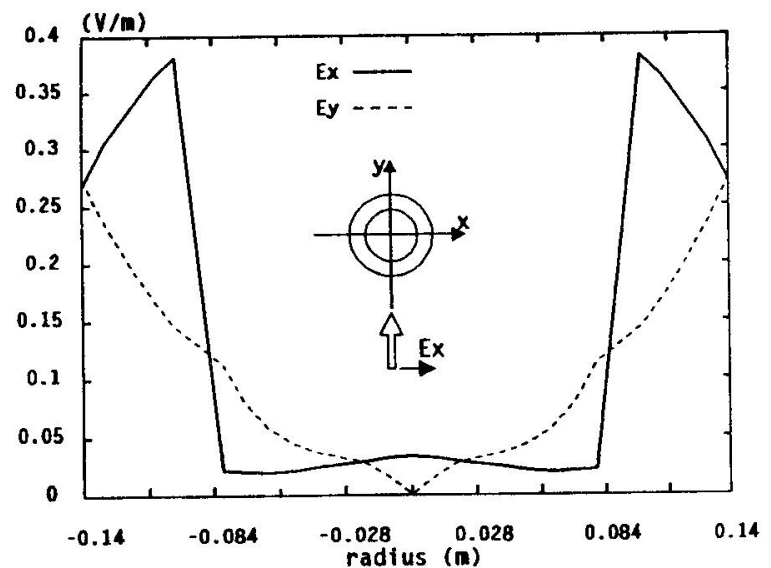

Fig. $3 \mathrm{X}$-axis cut of the electric field in the TE case.

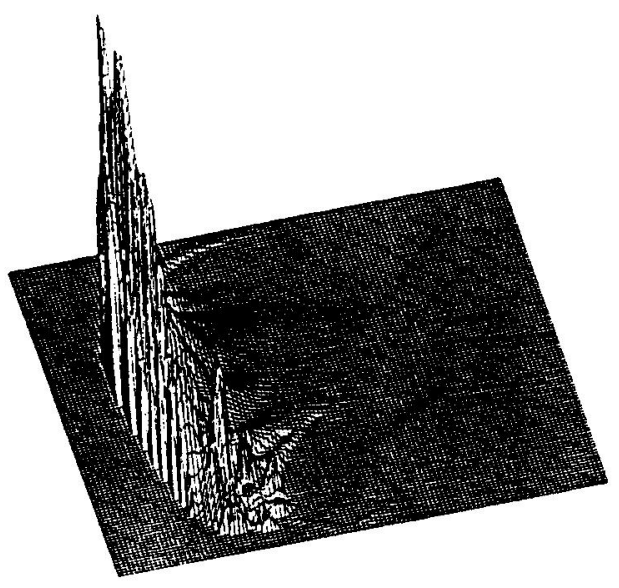

Fig. 5 Bidimensional plot of calculated SAR distribution into head section. Maximum value is $1.8310^{-3} \mathrm{~W} / \mathrm{Kg}$.

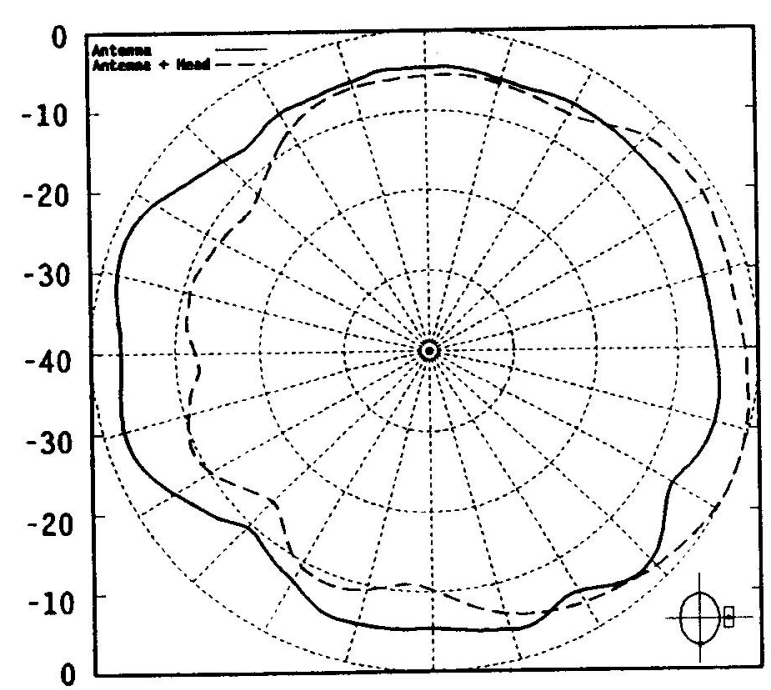

Fig. 7 Measured diagram of a short monopole alone and with the head phantom.

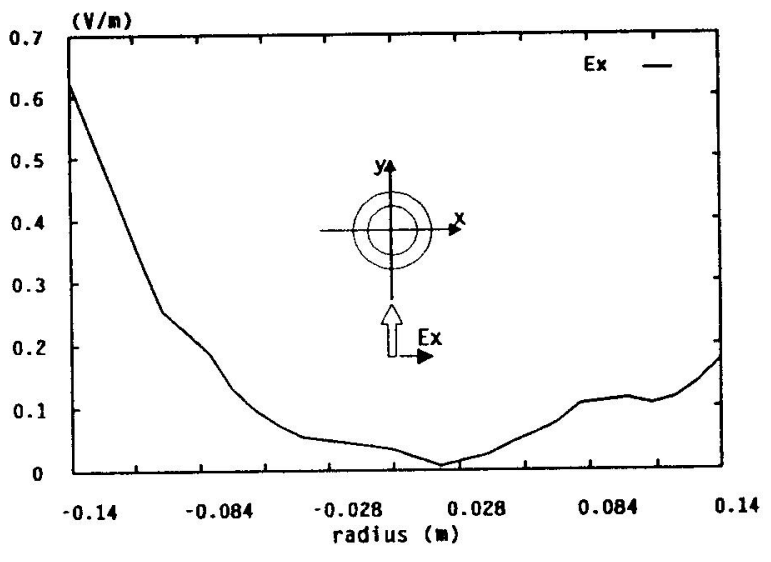

Fig. 4 Y-axis cut of the electric field in the TE case.

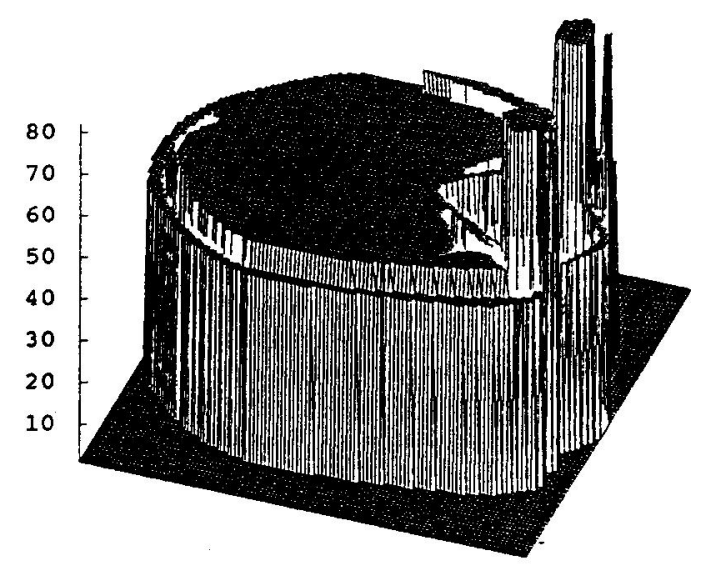

Fig. 6 Numerical model of the head. Threedimensional plot shows the modulus of its tissue permittivity. Eyes are the two highest points.

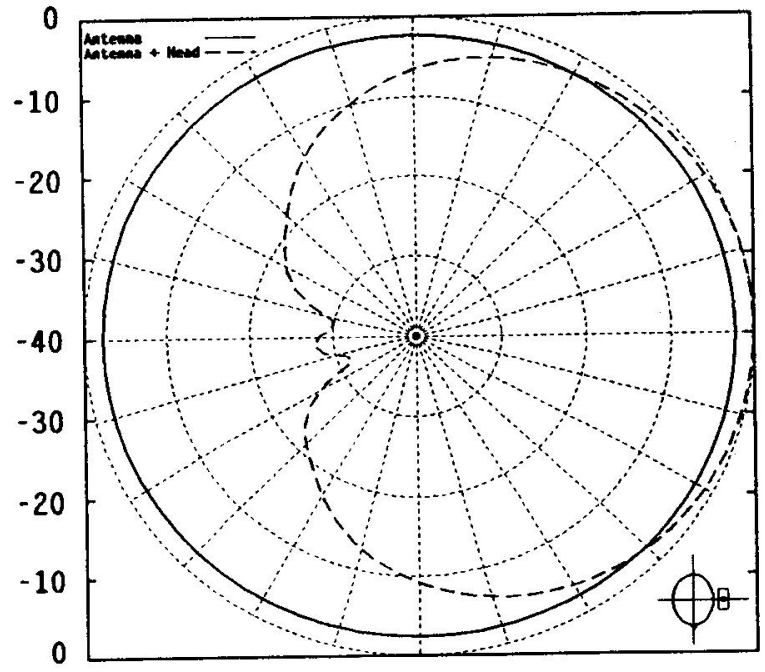

Fig. 8 Computed radiation pattern of short monopole. 